\title{
Therapeutic effect of high-efficiency online hemodiafiltration for recurrent restless legs syndrome in dialysis patients
}

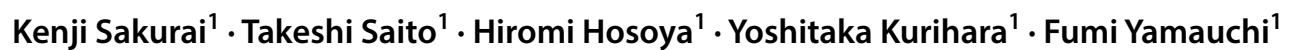

Received: 27 August 2019 / Accepted: 17 March 2020 / Published online: 30 March 2020

(c) The Author(s) 2020

\begin{abstract}
Two dialysis patients developed recurrent restless legs syndrome. The clinical courses and the association between the $\alpha_{1}$ microglobulin removal rate and the therapeutic effects of hemodiafiltration were analyzed. Case 1: a middle-aged woman was switched from predilution online hemodiafiltration to hemodialysis, following which the $\alpha_{1}$-microglobulin removal rate decreased from 39.1 to $29.9 \%$. A month later, the severe restless legs syndrome occurred. The treatment was then switched to high-efficiency hemodiafiltration and 2 weeks later, these symptoms were resolved. The $\alpha_{1}$-microglobulin removal rate increased to $41.9 \%$. Her symptoms recurred 5 years later with severity; thus, the hemodiafiltration treatment conditions were changed. Under revised conditions, the $\alpha_{1}$-microglobulin removal rate was $42.6 \%$, and her symptoms were alleviated. Continuation of high-efficiency hemodiafiltration led to the resolution of the syndrome at 1 month after recurrence. Case 2: a middle-aged man on hemodialysis developed the restless legs syndrome in the second year of treatment. The $\alpha_{1}$-microglobulin removal rate was $23.8 \%$. After switching to a month-long high-efficiency hemodiafiltration with a removal rate of $\geq 40 \%$, his symptoms were resolved. However, the syndrome recurred after a year with severity. The symptoms were alleviated using various measures. The hemodiafilters were changed, and hemodiafiltration with an $\alpha_{1}$-microglobulin removal rate of $\geq 40 \%$ was continued; 2 months later, his symptoms resolved. High-efficiency online hemodiafiltration is an effective therapeutic strategy for restless legs syndrome in dialysis patients. We found, for the first time, that target removal efficiency is an $\alpha_{1^{-}}$ microglobulin removal rate of $40 \%$ or higher.
\end{abstract}

Keywords Hemodiafiltration $\cdot \alpha_{1}$-microglobulin $\cdot$ Restless legs syndrome $\cdot$ Albumin leakage

\section{Introduction}

Restless legs syndrome (RLS) is mainly divided into primary (idiopathic) and secondary (symptomatic) RLS. The development of primary RLS has not been fully elucidated; however, a dopaminergic neuron hypofunction, the relationship between iron metabolism and dopamine, and genetic predisposition are considered as risk factors for RLS. Secondary RLS is considered to be caused by other diseases or

Electronic supplementary material The online version of this article (https://doi.org/10.1007/s10047-020-01164-1) contains supplementary material, which is available to authorized users.

Kenji Sakurai

ssakurai@ peach.ocn.ne.jp

1 Hashimoto Clinic, 3-21-5 Hashimoto Midori-ku, Sagamihara, Kanagawa 252-0143, Japan drugs. In particular, the incidence rate is high in pregnant women, patients with iron deficiency anemia, patients with diabetes mellitus and patients with end stage renal disease $[1,2]$. The causative substances of RLS in dialysis patients have not been elucidated yet, and no detailed investigation has been performed.

We previously reported that the RLS symptoms in dialysis patients improved through the use of online hemodiafiltration (OL-HDF), which increased the removal efficiency of the low molecular weight proteins (LMWP) [3].

In this study, we aimed to describe the clinical course of two patients with recurrent RLS and investigate the therapeutic effect of high-efficiency OL-HDF. 


\section{Case presentation}

Case 1: a female patient in her 50s with polycystic kidney disease (primary disease) and a 12-year history of dialysis at the time of recurrence.

Her condition was stable during predilution online HDF (pre-HDF) using FDY-180GW (PEPA membrane, Nikkiso Co., Ltd). Although OL-HDF was approved by the health insurance (in 2010, indicated for dialysis amyloidosis and disdialysis syndrome), it was not indicated for the pathological condition of this patient. Thus, the method was changed to hemodialysis (HD). The removal rates of $\beta_{2}$-microglobulin (MG) and $\alpha_{1}$-MG were $83.1 \%$ and $82.0 \%$, respectively, for HDF and $39.1 \%$ and $29.9 \%$, respectively, for HD. While there was a slight change in the $\beta_{2}$-MG removal rate following the switch to $\mathrm{HD}$, the $\alpha_{1}$-MG removal rate decreased by 9 points. However, Kt/V for urea increased from 1.8 to 2.3. RLS developed approximately 1 month after the switch from OL-HDF to HD. Symptoms were very severe, with an international restless legs syndrome study group severity scale (IRLS) score of 32 [4].

The patient was switched to a high-performance dialyzer and continued to receive longer HD sessions (from 4 to $4.5 \mathrm{~h}$ ) following which, the RLS symptoms gradually subsided.
However, the patient was not completely cured and complained of shoulder joint pain and severe insomnia. Therefore, the dialysis method was changed to $50 \mathrm{~L} / \mathrm{session}(\mathrm{s}$ ) preHDF; RLS resolved 2 weeks after the change. At the time of RLS resolution, the removal rates of $\beta_{2}-\mathrm{MG}$ and $\alpha_{1}$-MG were $85.9 \%$ and $41.9 \%$, respectively, (Fig. 1). Changes in the $\alpha_{1}-$ MG removal rate $(39.1 \% \rightarrow 29.9 \% \rightarrow 41.9 \%)$ correlated with the symptoms during this episode (stabilization $\rightarrow$ worsening $\rightarrow$ alleviation). Subsequently, high-efficiency pre-HDF $\left(\beta_{2}\right.$-MG removal rate $\geq 80 \%, \alpha_{1}$-MG removal rate $\geq 35 \%$ ) was continued.

RLS recurred in this patient 5 years later. Since the symptoms were severe with an IRLS score of 28 , the total substitution fluid (total Qs) for the pre-HDF (blood flow rate [Qb]: $250 \mathrm{~mL} / \mathrm{min}$ ) was increased from 60 to $65 \mathrm{~L} / \mathrm{s}$ using MFX-19U (PES membrane, Nipro). The treatment time was extended from 4 to $4.5 \mathrm{~h}$. In the third efficiency evaluation after the change in HDF parameters, the removal rates of $\beta_{2^{-}}$ MG and $\alpha_{1}-\mathrm{MG}$ were found to be $85.9 \%$ and $42.6 \%$, respectively, and the IRLS score improved to 21 on the 9th day after recurrence. However, RLS symptoms persisted, and the method was switched back to $50 \mathrm{~L} / \mathrm{s}$ pre-HDF using GDF21 (PEPA membrane, Nikkiso Co.,Ltd) for further highefficiency HDF. The removal rate of $\alpha_{1}$-MG was $48.4 \%$, its
Fig. 1 The figure shows the initial onset and the course of RLS in cases 1 and 2. The patient in case 1 was slightly nervous, but the dialysis course was stable with HDF. However, RLS developed 1 month after switching from HDF to HD. The dialysis efficiency of HD was increased, and though RLS was alleviated, it was not resolved. Thus, the method was switched to HDF. In Case 2, RLS developed in the 2nd year of dialysis and, thus, primary RLS was suspected. However, high-efficiency HDF was performed and the patient recovered after 1 month of the entire course

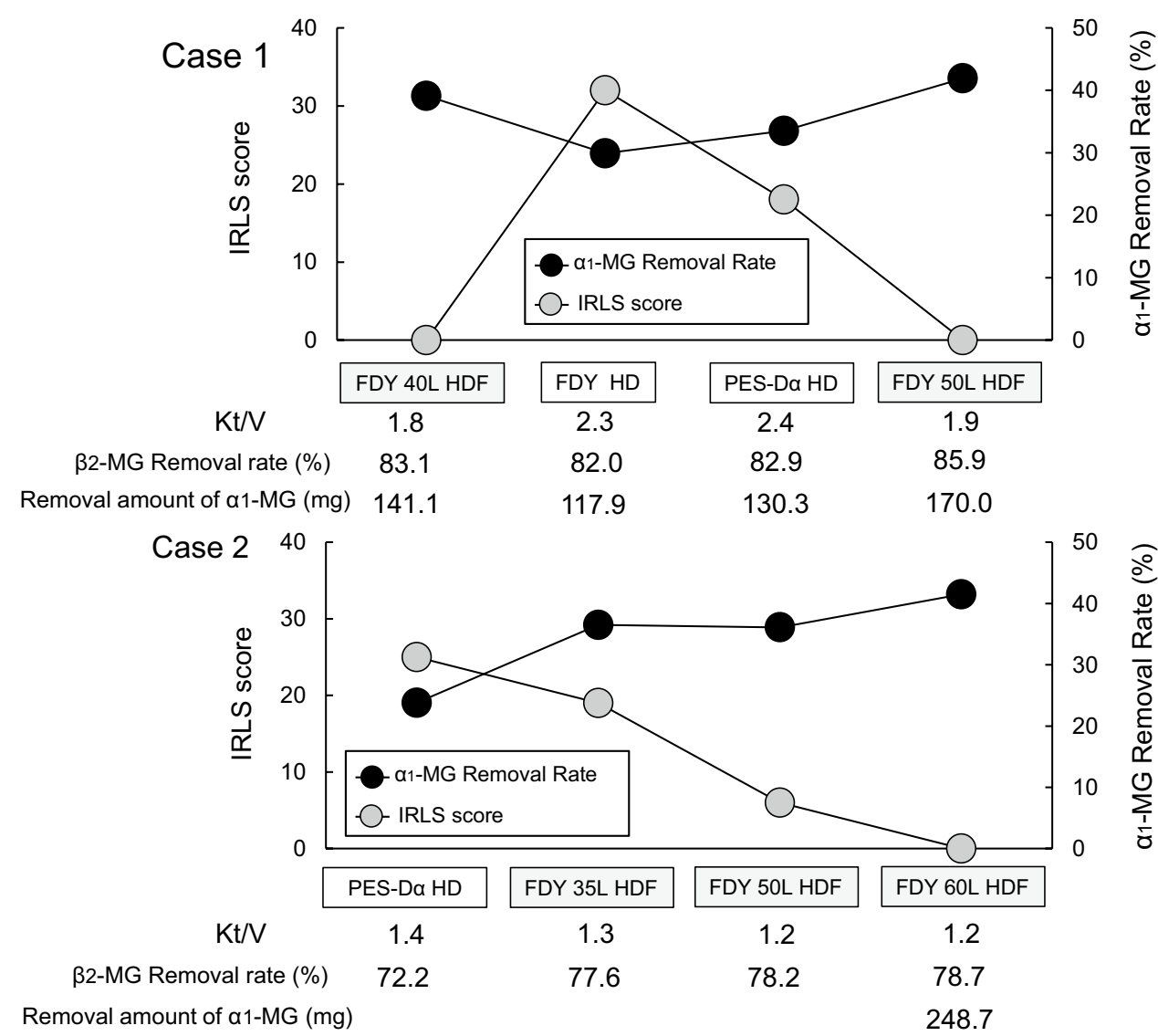


removal amount was $190 \mathrm{mg}$, and the IRLS score improved to 10 .

RLS was resolved 1 month after recurrence by continuing pre-HDF with the $\alpha_{1}$-MG removal rate of $\geq 45 \%$. The serum albumin (Alb) level was $3.6 \mathrm{~g} / \mathrm{dL}$ before recurrence, but decreased to $3.3 \mathrm{~g} / \mathrm{dL}$ with an increase in the amount of Alb leakage. Therefore, after the RLS symptoms disappeared, the total Qs was decreased to $45 \mathrm{~L} / \mathrm{s}$. Since the recovery of serum Alb level was delayed, 1 month after the patient was cured, the condition was changed to $50 \mathrm{~L} / \mathrm{s}$ preHDF using FIX-210Seco (ATA membrane, Nipro). Under these conditions, the removal rate of $\alpha_{1}$-MG was $38.0 \%$ with a removal amount of $144 \mathrm{mg}$, and the amount of Alb leakage was $2.6 \mathrm{~g} / \mathrm{dL}$. The serum Alb level increased to $3.6 \mathrm{~g} /$ dL 2 weeks after switching to FIX. RLS recurred with mild symptoms (IRLS score 5). When the total Qs was increased to $70 \mathrm{~L} / \mathrm{s}$, the removal rate of $\alpha_{1}$-MG became $39 \%$ with a removal amount of $158 \mathrm{mg}$. RLS symptoms disappeared 10 days later (Fig. 2).

Case 2: the patient was a man in his $40 \mathrm{~s}$, with a 4-year history of dialysis at the time of recurrence. While the patient was stable on HD, the first episode of RLS occurred 2 years after the start of dialysis. At the onset of RLS, the removal rates of $\beta_{2}$-MG and $\alpha_{1}$-MG were $72.2 \%$ and $23.8 \%$, respectively. The IRLS score improved from 25 to 6 after switching to pre-HDF using FDY-GW with an $\alpha_{1}$-MG removal rate of $36 \%$. Since the symptoms persisted, the total Qs was increased from 50 to $60 \mathrm{~L} / \mathrm{s}$. An $\alpha_{1}$-MG removal rate of $\geq 40 \%$ was achieved and the HDF continued. RLS resolved after 1 month of the entire course (Fig. 1).
Following RLS resolution, $64 \mathrm{~L} / \mathrm{s}$ pre-HDF $(\mathrm{Qb}$ : $250 \mathrm{~mL} / \mathrm{min}$, treatment time: $4 \mathrm{~h}$ ) was continued using MFX-25U. The patient sometimes complained of a mild abnormal sensation in the lower extremities; RLS recurred 1 year after healing. The symptoms were very severe with an IRLS score of 38. The performance of MFX-U deteriorated, and the removal rates of $\beta_{2}$-MG and $\alpha_{1}$-MG were $76 \%$ and $29 \%$, respectively, in the pre-HDF, when the total Qs was $64 \mathrm{~L} / \mathrm{s}$ and $\mathrm{Qb}$ was $300 \mathrm{~mL} / \mathrm{min}$. The treatment was strengthened by increasing the total Qs and extending the treatment time, without changing the hemodiafilter; however, an $\alpha_{1}$-MG removal rate of $\geq 35 \%$ could not be obtained. Thus, the method was changed to postdilution online HDF (post-HDF) with a total Qs of $20 \mathrm{~L} / \mathrm{s}$; the $\alpha_{1}$-MG removal rate was $35 \%$. Although the IRLS score improved to 5, the RLS was not resolved. Thus, the treatment was switched to pre-HDF using FDY-GW, with the total Qs of $60 \mathrm{~L} / \mathrm{s}$. An $\alpha_{1}$-MG removal rate of $\geq 40 \%$ was achieved. Although the amount of Alb leakage was excessive, this HDF treatment was continued and RLS resolved approximately 2 months after the recurrence (Fig. 3).

RLS recurred the following year (IRLS score 20) after two conditions of low-efficiency HDF were implemented (the removal rate of $\alpha_{1}$-MG: 1 st $25 \%, 2$ nd $26 \%$ ). The treatment was thus revised to $50 \mathrm{~L} / \mathrm{s}$ pre-HDF (using GDF21, Qb: $300 \mathrm{~mL} / \mathrm{min}$ ) and $4.5 \mathrm{~h}$ of treatment time; RLS resolved 3 weeks after the recurrence. Under these conditions, the removal rates of $\beta_{2}$-MG and $\alpha_{1}$-MG were $83.1 \%$ and $41.4 \%$, respectively, and the amount of Alb leakage was $4.2 \mathrm{~g}$.
Fig. 2 The figure shows the course at the time of recurrence in case 1 . There was no apparent decrease in dialysis efficiency, but RLS recurred. After the recurrence, HDF was performed with an $\alpha_{1}$-MG removal rate of $\geq 40 \%$. Alb leakage increased and the serum Alb level decreased, but RLS was resolved. The removal rate and amount of $\alpha_{1}$-MG at the time of RLS resolution after the initial onset were $41.9 \%$ and $170 \mathrm{mg}$, respectively, and after recurrence were $54.5 \%$ and $234 \mathrm{mg}$, respectively. This indicates that higher-efficiency $\mathrm{HDF}$ was required at the time of recurrence

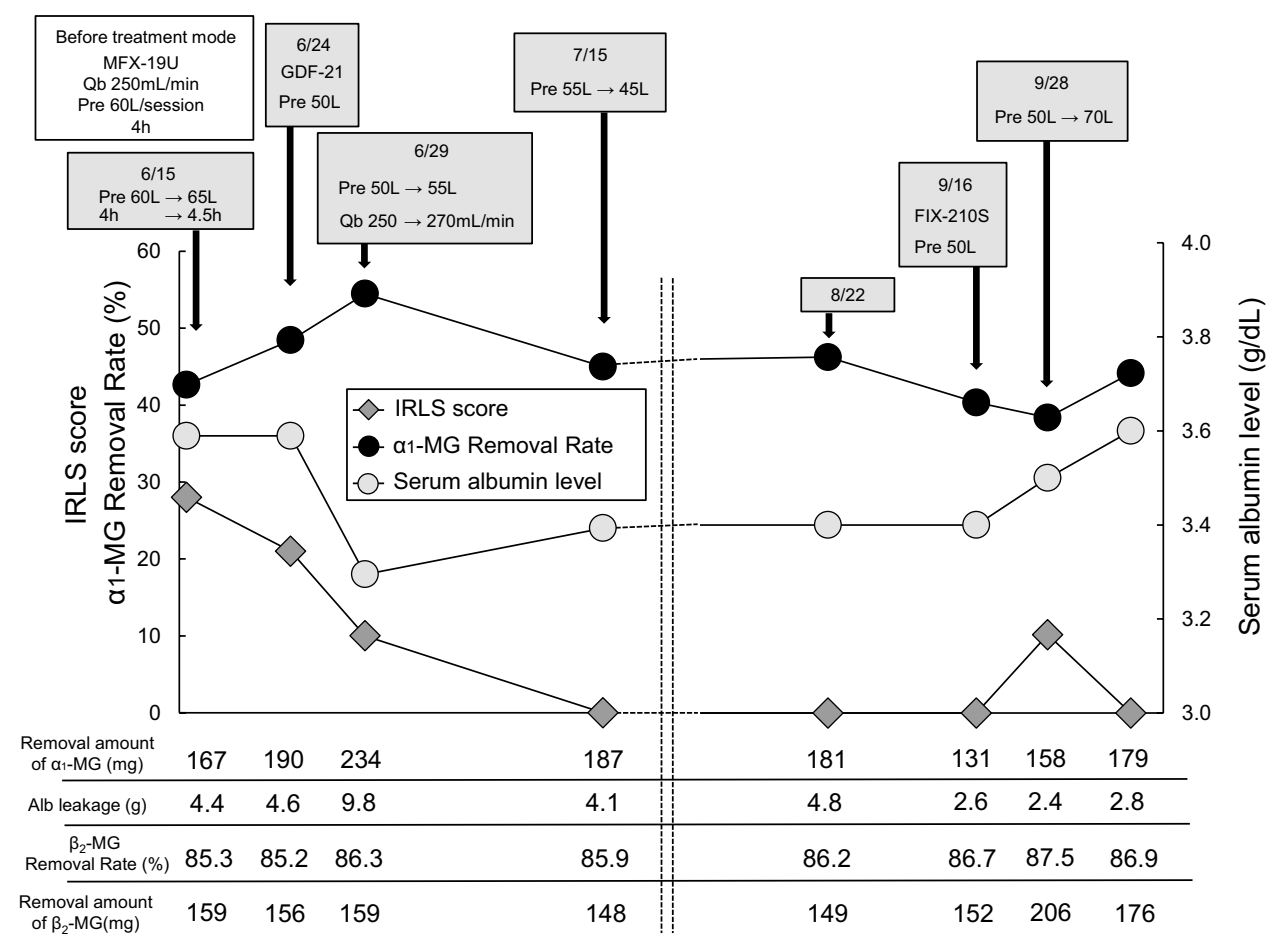


Fig. 3 Course at the time of recurrence in case 2 . It is strongly suspected that recurrent RLS was caused by deterioration of the hemodiafilter, MFXU. Even when the total Qs was increased or when post-HDF was performed, the removal rate of $\alpha_{1}$-MG did not reach $40 \%$, which caused a delay in RLS resolution. The patient was successfully treated by FDY-GW with a large pore size. In this patient, the removal rate and amount of $\alpha_{1}-\mathrm{MG}$ at the time of RLS healing after the initial onset were $41.5 \%$ and $248 \mathrm{mg}$, respectively, and after recurrence were $43.6 \%$ and $278 \mathrm{mg}$, respectively

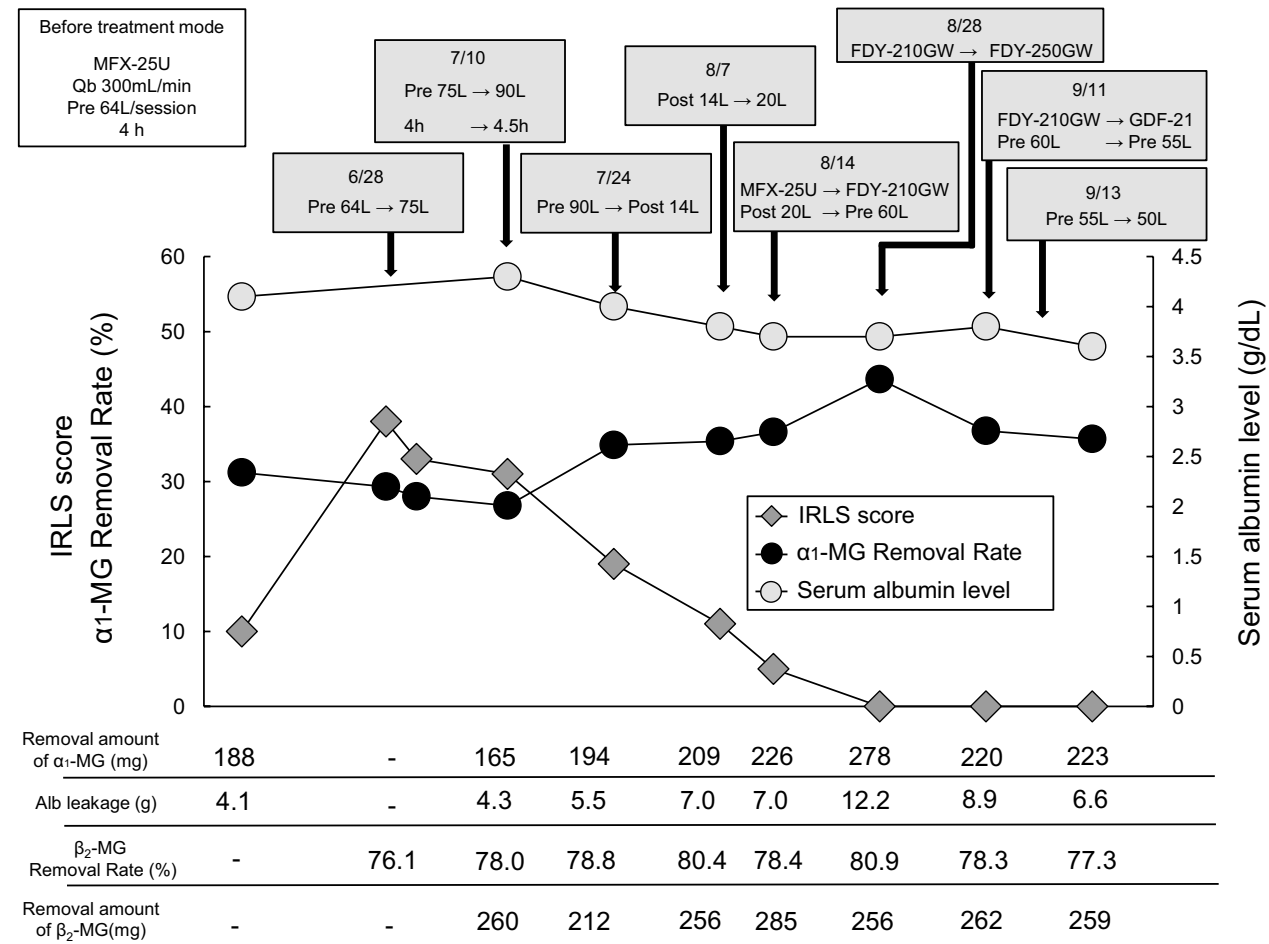

During the observation period, no obvious iron deficiency was observed in both patients.

Informed consent for publication of this report was obtained from both patients included in this study.

\section{Discussion}

The International Restless Legs Syndrome Study Group provides diagnostic criteria for RLS, and the criteria for severity classification have also been established $[4,5]$. The severity is classified into four grades as follows: mild (score, 0-10), moderate (score, 11-20), severe (score, 21-30), and very severe (score, 31-40).

Previous studies showed that RLS occurred in $12 \%$ to $62 \%$ of dialysis patients [6-9], and higher incidence rates of RLS among dialysis patients have been reported in studies outside Japan. This may be attributable to the fact that for dialysis therapy in Japan, ultra-pure dialysis fluids and highor super high-flux dialyzers with excellent biocompatibility are frequently used, which ensure the quality of dialysis to be satisfactory. At our clinic, we encountered nine patients with RLS over the past 10 years (2010-2019). One patient was transferred to another facility, and the clinical course is thus unknown. In the other eight patients, RLS was resolved by high-efficiency HDF. At the time of writing this report (December 2019), 119 patients were receiving maintenance dialysis at our clinic, none of whom had RLS. In addition, none of the patients experienced a new onset of RLS during the last 5 years.

According to our clinical experience, RLS was observed among patients receiving maintenance $\mathrm{HD}$, patients in whom OL-HDF therapy was switched to conventional HD therapy, and patients in whom $\alpha_{1}$-MG removal efficiency decreased due to some causes even during OL-HDF therapy.

At the time of recurrence of RLS in case 1, the manufacturer of the hemodiafilter was requested to explain whether the performance level of the product would decrease at the time of shipment of the hemodiafilter of that particular lot. The person in charge replied that the pore size of the dialysis membrane was within the reference range, although it was at the minimum acceptable limit. Accepting their reply, we took this into consideration regarding the cause of the onset of RLS; however, the removal efficiency of OL-HDF immediately before RLS recurrence in this patient was not evaluated at our clinic, so that the cause of the RLS recurrence remains unknown.

We infer that the RLS recurrence in case 2 was caused by the decreased performance level of the hemodiafilter. High-efficiency hemodiafilters with decreased removal performance levels might be supplied, causing a change in the patient's clinical response. According to the lot of highefficiency hemodiafilters, the removal performance levels slightly differed for $\beta_{2}$-MG and greatly differed for $\alpha_{1}-\mathrm{MG}$ (Fig. 4).

While the cause of RLS in dialysis patients has not been established, some types of uremic toxin with a molecular 
Fig. 4 The same product from the same brand may differ in efficiency depending on the production date. To detect this difference, $\alpha_{1}$-MG removal rate and Alb leakage should be measured. If the evaluation is only for $\beta_{2}-\mathrm{MG}$, all products would show the same efficiency

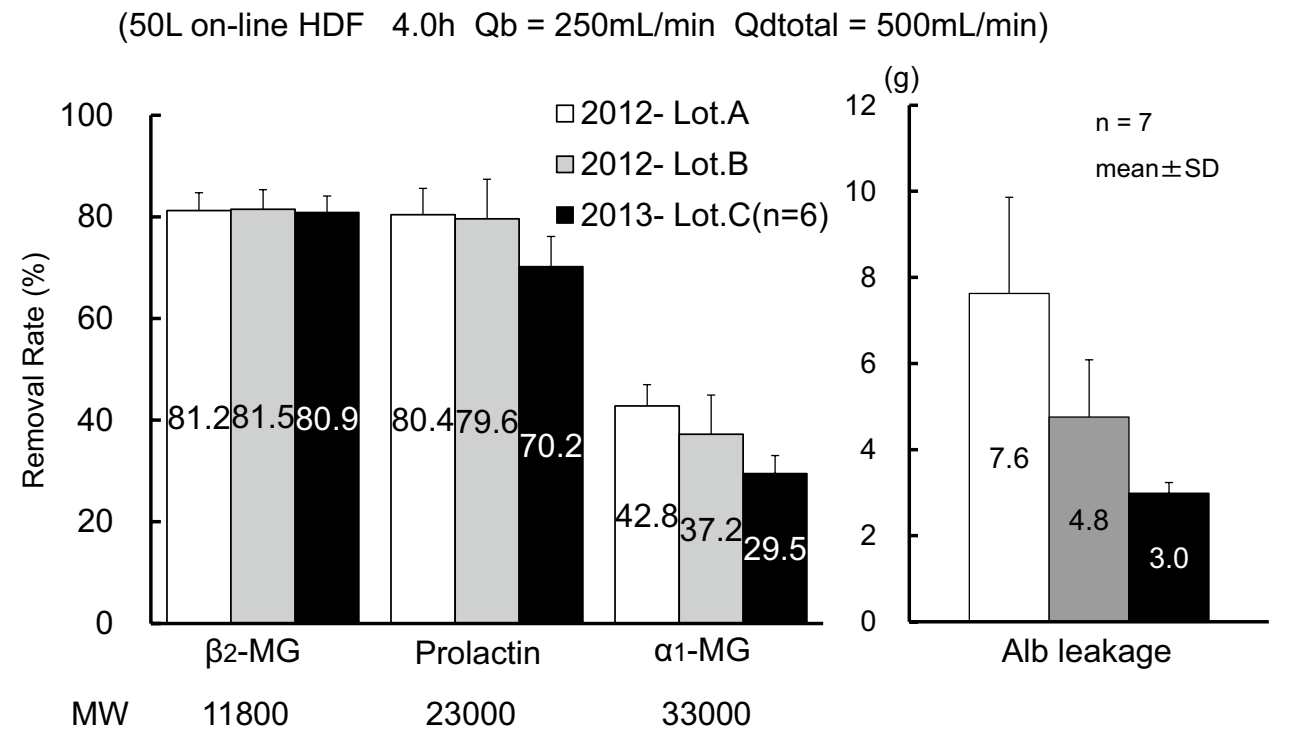

weight greater than that of $\beta_{2}-\mathrm{MG}$ or protein-bound toxin are considered causative substances [10]. We consider that the accumulation of some types of uremic toxins may cause functional abnormalities (or inflammation) of peripheral nerves, thereby causing RLS. In addition, $\alpha_{1}-\mathrm{MG}$ has been reported to have an antioxidant property [11-13]. If it is assumed that RLS is caused by chronic inflammation of peripheral nerves due to oxidative stress, the significance of efficient removal of $\alpha_{1}$-MG can be understood. High efficient removal of $\alpha_{1}$-MG promotes the turnover of $\alpha_{1}$-MG by removing $\alpha_{1}$-MG which has lost its antioxidant property and promoting the generation of antioxidant $\alpha_{1}$-MG in the liver. This mechanism may lead to recovery from RLS. Higuchi et al. reported that blood levels of $8-\mathrm{OHdG}$, an oxidative stress marker, were higher in dialysis patients with RLS than in dialysis patients without RLS, and that blood levels of 8-OHdG correlated with the severity of RLS. The authors suggested an involvement of oxidative stress in the pathogenesis of RLS in dialysis patients [9].

From the above-mentioned perspective, high-efficient removal of $\alpha_{1}$-MG is essential for the treatment of RLS in dialysis patients for the following reasons: (1) direct removal of causative substances categorized in larger LMWPs; (2) increased removal of protein-bound toxins by increasing the amount of Alb leakage; and (3) removal of old non-antioxidant $\alpha_{1}$-MG to increase synthesis of new $\alpha_{1}$-MG in the liver.

For dialysis patients with RLS, aggressive treatment with constant high-efficiency HDF that can achieve an $\alpha_{1}$-MG removal rate of at least $40 \%$ should be performed after assessment for iron deficiency. Although excessive Alb leakage cannot be avoided in high-efficiency HDF, the amount of Alb leakage of up to approximately 8-9 g should be set as an acceptable level in the early phase of treatment to promptly improve the patient's quality of life. In case 2 in this report, in addition to the increase in $\alpha_{1}$-MG removal rate, the protein-bound toxins were successfully removed by setting the amount of Alb leakage to $5.5 \mathrm{~g} / \mathrm{s}$ or more, which may have resulted in the cure of RLS.

Specific conditions are as follows: treatment with preHDF using GDF, MFX-U, or FIX-U should be started with a Qb of $250-300 \mathrm{~mL} / \mathrm{min}$, total Qs of $50 \mathrm{~L} / \mathrm{s}$, and treatment time of $4 \mathrm{~h}$. Treatment with post-HDF using FIX$\mathrm{S}$, ABH-PA, or NVF-H should be started with a $\mathrm{Qb}$ of $250-300 \mathrm{~mL} / \mathrm{min}$, total Qs of $12 \mathrm{~L} / \mathrm{s}$, and treatment time of $4 \mathrm{~h}$. During HDF therapy, therapeutic effects should be confirmed by examinations for $\alpha_{1}$-MG removal rates and amounts, and amounts of Alb leakage and high-efficiency HDF should be continued under appropriate treatment conditions. Extension of treatment time contributes to shortening of treatment duration.

If the therapeutic effect is not improved even with highefficiency HDF, an excessively higher removal rate of $\alpha_{1}$ MG, as obtained by hematocrit correction, may be indicated. The removal amount of $\alpha_{1}$-MG should be examined in consideration of any dissociation between its removal rate and removal amount. This can occur when plasma refilling is slow or when blood recirculates within the blood circuit. In such cases, the treatment conditions of HDF should be set with a target $\alpha_{1}$-MG removal amount of $180 \mathrm{mg}$ or, when possible, $200 \mathrm{mg}$.

With the same $\alpha_{1}$-MG removal efficiency, no superiority or inferiority in clinical effects was observed between the two treatment modes of pre- and post-HDF. We consider that OL-HDF should be continued for 1 month after RLS resolution under the dialysis conditions at the time of resolution. 


\section{Conclusion}

Two cases of recurrent RLS in dialysis patients are presented in this study. High-efficiency online HDF is an effective therapeutic strategy for severe or very severe RLS (IRLS score: 21 or more) in dialysis patients, having reached the target removal efficiency in terms of $\alpha_{1}$-MG removal rate of $40 \%$ or higher and $\alpha_{1}$-MG removal amount of $200 \mathrm{mg}$.

Open Access This article is licensed under a Creative Commons Attribution 4.0 International License, which permits use, sharing, adaptation, distribution and reproduction in any medium or format, as long as you give appropriate credit to the original author(s) and the source, provide a link to the Creative Commons licence, and indicate if changes were made. The images or other third party material in this article are included in the article's Creative Commons licence, unless indicated otherwise in a credit line to the material. If material is not included in the article's Creative Commons licence and your intended use is not permitted by statutory regulation or exceeds the permitted use, you will need to obtain permission directly from the copyright holder. To view a copy of this licence, visit http://creativecommons.org/licenses/by/4.0/.

\section{References}

1. Akbom KA. Restless legs: a report of 70 new cases. Acad Med Scand. 1950;246(Suppl):64-8.

2. Suzuki K, Ohida T, Sone T, et al. The prevalence of restless legs syndrome among pregnant women in Japan and relationship between restless legs syndrome and sleep problems. Sleep. 2003;26(6):673-7.

3. Sakurai K. Biomarkers for evaluation of clinical outcomes of hemodiafiltration. Blood Purif. 2013;35(Suppl 1):64-8.

4. Allen RP, Picchietti D, Hening WA, Trenkwalder C, Walters AS, Montplaisi J, Restless Legs Syndrome Diagnosis, and Epidemiology workshop at the National Institutes of Health; International Restless Legs Syndrome Study Group. Restless legs syndrome: diagnostic criteria, special considerations, and epidemiology: a report from the restless legs syndrome diagnosis and epidemiology workshop at the National Institutes of Health. Sleep Med. 2003;4(2):101-19.

5. Walters AS, LeBrocq C, Dhar A, Hening W, Rosen R, Allen RP, Trenkwalder C, International Restless Legs Syndrome Study Group. Validation of the International Restless Legs Syndrome Study Group rating scale for restless legs syndrome. Sleep Med. 2003;4(2):121-32.

6. La Manna G, Pizza F, Persici E, Baraldi O, Comai G, Cappuccilli ML, Centofanti F, Carretta E, Plazzi G, Colì L, Montagna P, Stefoni S. Restless legs syndrome enhances cardiovascular risk and mortality in patients with end-stage kidney disease undergoing long-term haemodialysis treatment. Nephrol Dial Transplant. 2011;26(6):1976-83.

7. Banno M, Otsubo S, Takagi M, Sugimoto H, Nitta K. Relationship between restless legs syndrome and mortality in hemodialysis patients. Nihon Toseki Igakkai Zasshi. 2015;48(1):45-50.

8. Lin XW, Zhang JF, Qiu M-Y, Ni L-Y. Restless legs syndrome in end stage renal disease patients undergoing hemodialysis. BMC Neurol. 2019;19(1):47.

9. Higuchi T, Abe M, Mizuno M, Yamazaki T, Suzuki H, Moriuchi M, Oikawa O, Okawa E, Ando H, Okada K. Association of restless legs syndrome with oxidative stress and inflammation in patients undergoing hemodialysis. Sleep Med. 2015;16(8):941-8.

10. Shinzato T, Maeda K. Push/pull hemodiafiltration. Contrib Nephrol. 2007;158:169-76.

11. Åkerström B, Maghzal GJ, Winterbourn CC, Kettle AJ. The lipocalin $\alpha 1$-microglobulin has radical scavenging activity. J Biol Chem. 2007;282(43):31493-503.

12. Åkerström B, Lögdberg L. $\alpha 1$-Micoroglbulin Madame Curie bioscience database. Austin (TX): Landes Bioscience; 2000-2003

13. Satin M, Cannas M. Collagen-bound $\alpha 1$-microglobulin in normal and healed tissues and its effect on immunocompetent cells. Scand J Immunol. 1999;50:289-95.

Publisher's Note Springer Nature remains neutral with regard to jurisdictional claims in published maps and institutional affiliations. 